\title{
Editorial
}

\section{KANELLOPOULOS-KERATOCONUS DIAGNOSIS AND TREATMENT, 2014}

\section{A Few Thoughts, Unanswered Questions and Hopes in regard to Keratoconus Diagnosis and Treatment}

As the corneal collagen cross-linking (CXL) treatment is intended to be applied in eyes developing progressive ectasia, it is of paramount importance the establishment of early and sensitive criteria for the diagnosis of keratoconus as well as the establishment of possible progression.

\section{Keratoconus Assessment, Need for Development of Updated Keratoconus Criteria}

There are various keratoconus diagnosis, staging and progression criteria that are currently in clinical use (albeit some of them of narrow value). Among them, there are different clinical and topographic/topometric measurements. Clinical measurements include visual acuity [uncorrected distance visual acuity (UDVA) and best-spectacle corrected distance visual acuity (CDVA)] as well as manifest refraction. Topographic/topometric measurements (quantitative and qualitative) include corneal keratometry, anterior/posterior elevation, curvature asymmetry, corneal pachymetry and so on. Combinations of these parameters are incorporated in various decision-tree schemes or staging classification systems, such as the Rabinowitz, ${ }^{1}$ Klyce, ${ }^{2}$ and Amsler-Krumeich criteria. ${ }^{3}$

Our long clinical experience with keratoconus screening and management (as has been reported and published in the peer-review literature) indicates, e.g. that corneal pachymetry and visual acuity may not be very reliable indicators of ectasia or keratoconus progression assessment. Reduced visual acuity per se may not correlate with the severity of keratoconus and may only be presented in very advanced stages of the disease. Published results from our team indicate that the assessment of keratoconus severity and visual function has yielded poor results in a number of anterior surfacederived topographic parameters (including keratometry, pachymetry, surface-asymmetry indices) in keratoconic eyes. ${ }^{4}$ Published results from other investigators also indicate the specificity and sensitivity limitations of the traditionally employed keratoconus criteria in clinical use. ${ }^{5-7}$

In addition, despite the use of several topographic/topometric diagnostic criteria, clinicians in everyday practice often face cases that cannot be fully fitted either in the keratoconus or in the 'normal cornea' groups. These suspect cases represent a real diagnostic and management challenge for the clinician. This further emphasizes the need for research to narrow the limitations of the currently used criteria. ${ }^{3,8}$

The refinement and augmentation of early diagnostic criteria for keratoconus is important, because it may enable timely disease management and, therefore, offer better outcomes for the keratoconic patients.

\section{The Need for Evaluation of CXL Protocols}

Over the last 10 years, CXL has evolved to be a significant innovation for the arrest of the progression of keratoconus. Since the original Dresden protocol ( $3 \mathrm{~mW} / \mathrm{cm}^{2}$ for $\left.30 \mathrm{mins}\right),{ }^{9}$ several treatment CXL protocol variations have been introduced, most of them by our team (REFS). These, however, have not been fully correlated as far as their efficacy and safety is concerned. These variations involve higher fluence, such as the use of $6,10,18$ and $30 \mathrm{~mW} / \mathrm{cm}^{2}$, and correspondingly shorter UV-A exposure time, aiming to deliver the same amount of energy (usually $5.4 \mathrm{~J} / \mathrm{cm}^{2}$ ), and presumably the same stiffening effect. While several assessment modalities exist, including ex vivo biomechanical (tensile strength), ${ }^{10}$ biochemical (enzymatic digestion) $)^{11}$ or in vivo methods [e.g. via optical coherence tomography (OCT) imaging-derived analysis], ${ }^{12}$ to the best of our knowledge, so far no direct comparative study of these CXL protocols has been presented.

\section{Current Treatment Options or Tools/Methods Currently in Use}

\section{Current Methods for the Diagnosis of Keratoconus}

Keratoconus diagnosis has employed either clinical or topographic/topometric measurement-based criteria. Among them, keratometry, either traditionally obtained with keratometers, or, in the last 20 years, topography and/or topometry-based measurements, reported as simulated keratometries (SimK) have been employed. Such topography systems may be Placido, slit-scan topography or Scheimpflug imaging. Additionally, there is pachymetry (obtained either by ultrasound, slit-scan 
topography, or Scheimpflug imaging) as well as manifest refractive spherical equivalent (MRSE), measured subjectively. Limitations, however, do exist, e.g. in relation to measurement accuracy for current topographers, ${ }^{13}$ which is a problem compounded by corneal warpage in rigid contact lens wearers as well as pachymetry measurements by Scheimpflug imaging, ${ }^{14}$ which may be suspect to error when corneal clarity is compromised.

Some of the established criteria for keratoconus progression $v s$ baseline measurement used in contemporary clinical trials of CXL include the following: ${ }^{15-17}$

- $\mathrm{K}_{\max }$ (steepest keratometry) $\geq 1$ dioptres (D) increase

- $\mathrm{K}_{\max }-\mathrm{K}_{\min } \geq 1 \mathrm{D}$ increase $\left(\mathrm{K}_{\min }\right.$, flattest keratometry)

- $\mathrm{K}_{\text {mean }} \geq 0.75 \mathrm{D}$ increase $\left.\left[\mathrm{K}_{\text {mean }}=\left(\mathrm{K}_{\max }+\mathrm{K}_{\min }\right) / 2\right)\right]$

- Pachymetry $\geq 2 \%$ decrease in central corneal thickness

- Corneal apex power $\geq 1 \mathrm{D}$ increase (measured with cone location and magnitude index)

- MRSE change $\geq 0.5 \mathrm{D}$.

Several established decision trees exist based on combinations of the above, such as the Klyce indices of surface asymmetry index (SAI) and surface regularity index (SRI) and the KISA\% index. ${ }^{18}$

\section{Current Treatment Options for Keratoconus Management}

Up until 15 years ago, no interventions were available to arrest or decelerate disease progression. Keratoconus progression was traditionally managed with spectacle correction, until irregular astigmatism necessitated application of rigid gas permeable (RGB) contact lenses.

In cases when this was not possible or there was RGP intolerance (estimated up to $21 \%$ of keratoconic eyes), ${ }^{19}$ surgical procedures were also offered. Traditionally, a penetrating keratoplasty (PK), in which the central $2 / 3 \mathrm{~s}$ of the patient's cornea were discarded and substituted with a fresh donor cornea. ${ }^{20}$ This procedure is associated with significant morbidity as usually it takes about a week for the patient to return to normal everyday life and months, if not years before that eye can be adequately visually rehabilitated. It is noted that despite the use of this radical procedure, visual rehabilitation still necessitated an additional, refractive procedure on the corneal graft, in order to reduce the very common irregular astigmatism and high postoperative anisometropia associated with $\mathrm{PK} .^{21}$

Even in cases where PK generally achieved acceptable visual outcomes, long-term graft survival in keratoconic eyes declined rapidly after the 2 nd decade because the endothelial cells of the donor cornea tend to be slowly rejected by the host. Primary graft survival rates have been reported to $50 \%$ at 20 years, ${ }^{21,22}$ falling even further with repeat grafts.

An alternative to PK is deep anterior lamellar keratoplasty (DALK) which does not have the disadvantage of a PK short life span and associated complications. ${ }^{23}$ In DALK, this risk is possibly lower as the endothelial cell layer of the host is preserved: a median graft survival of 49 years for DALK vs 17 years for PK has been reported. ${ }^{24}$ It is noted, however, that DALK techniques are technically challenging.

Other treatment options (alternatives) for keratoconus are the insertion of intracorneal ring segments (ICRSs), which was pioneered in Europe (specifically in France). These PMMA inserts appear to significantly shift the shape of the cornea and are able to give significant visual rehabilitation. Their use has been quite widespread but there is no unison assessment of their efficacy. Many studies claim that the inserts have a significant number of complications associated with, as well as they are not always predictable and they may lose effect with time. Nevertheless, they too considered surgical procedure that needs to be repeated several times, as these rings may need to be substituted with larger or smaller rings and they may hamper the ability to assess the progression of keratoconus as they do distort the corneal surface into 'looking better.' In years to come, the clinician may lose the ability to assess the objective criteria of assessing keratoconus progression.

Collagen cross-linking, on the contrary, has shown repeatedly that it can effectively arrest the progression of keratoconus. The standard, epithelium-off Dresden protocol ${ }^{9}$ has been proven to be effective in arresting keratoconus progression. ${ }^{15-17}$ Data confirm a halt in ectatic progression by means of $\mathrm{K}_{\max }$ reduction, refraction stabilization, and CDVA improvement. ${ }^{25,26}$

Although generally safe, ${ }^{27}$ a range of complications has been reported after standard, epithelium-off CXL, including sterile corneal infiltrates, ${ }^{27}$ and significant risk of infectious keratitis, which appears to be the most significant complication.

In addition to the standard (Dresden) protocol CXL applications either epithelial (epi)-on (i.e. with no epithelial removal) or epi-off, the emergence of variations of high and very high-fluence CXL include both epi-on and epi-off, photorefractive intrastromal very high-fluence cross-linking epi-on and epi-off, as well as adjuvant prophylactic CXL along with routine LASIK. 
The efficacy of these cross-linking techniques may be assessed by postoperative visual rehabilitation, keratometry, and pachymetry stability measurements, as well as corneal rigidity measurements.

\section{Treatment Costs}

We have no access to objective data and numbers relating to the above question in regard to the European Union (EU) countries. Additionally, there will be a great range of these values from country to country. We attempt below to make an approximation based on our clinical and professional experience and some of the data published in the literature, although they mostly depict US data.

Regarding the treatment costs for the patient; briefly, RGB contact lenses use can cost anywhere from $€ 400$ to 600 per eye a year, a penetrating keratoplasty may cost: tissue, an average of $€ 2,000$ per eye, surgical material about $€ 2,000$, surgical fee from $€ 2,000$ to 5,000, and, of course, all the long-term follow-ups associated with a PK, which entails numerous office visits the 1st year, suture adjustments, refractions, contact lens fitting, a possible refractive procedure perhaps 2 years later, while a regraft on average about 15 years later is still within the realm of possibility.

The lifetime cost of conventional keratoconus treatment has been estimated at US \$25,168 per patient, with the likelihood of primary and repeat corneal transplantation being the key cost determinants. ${ }^{28}$ Given the relatively high prevalence of keratoconus and the frequency with which corneal transplantation is required, this represents a significant health economic burden. ${ }^{28}$ Noted, that most grafts for keratoconus were performed in relatively young patients.

Corneal transplantation is a costly intervention in advanced keratoconus [stage IV modified Krumeich classification, ${ }^{29}$ anterior $3 \mathrm{~mm}$ zone steep keratometry $(\mathrm{K})>55.5 \mathrm{D}$ ], with an estimated incremental cost-utility ratio of between $\$ 1,942^{30}$ and $\$ 3,025^{31}$ per quality-adjusted life-year. The life impact of repeat intervention, and slow, often incomplete, visual rehabilitation after transplantation should not be underestimated. ${ }^{32}$

The DALK procedure, although more laborios as a surgically procedure does carry similar costs with the PK, although the promise that it may not need to be repeated since the host endothelial cells are preserved. The ICRSs have estimated cost of about $€ 1,000$ for the rings, procedure materials about $€ 1,000$, surgical fee from $€ 1,000$ to 3,000, and again, all the follow-up adjustments and possible reoperations needed.

The costs of the cross-linking procedure vary greatly within the EU; they are estimated to be between $€ 1,500$ and 3,000 per eye, and it appears to be relatively stable procedure for the indication that it is used, meaning stabilization of ectasia. Very few reports of the need for a second CXL within many years and thousands of operations of experience, although in several cases a second refractive procedure may be mandated to visually rehabilitate these eyes that have been proven to be stable.

\section{Shortcomings}

The specific problems, treatment gaps and shortcomings of the current treatments that this proposal entails to fill, are as follows:

- No currently universally-accepted keratoconus diagnosis and progression criteria, as there are several empiric criteria used without any official directive by either the EU or the American Academy of Ophthalmology addressing the EU countries and the USA, and perhaps creating more elaborate and even more sensitive system may aid to the early diagnosis.

- The very important part of establishing progression of keratoconus that may command offering the specific patient population, the solution of collagen cross-linking.

- Besides the original Dresden protocol parameters evaluated in the most extensive way, all the newer-introduced CXL protocols do not carry the same weight in depth of ex vivo and in vivo evaluation. In short, efficacy correlation of the newer techniques has not been fully established.

Therefore, we do consider such evaluation to be mandated and we do consider the lack of this evaluation a significant shortcoming of these procedures, because they have become quite anecdotal: for example, no prospective randomized trial has ever compared them. So, we feel that there is a scientific lack of correlation of the collagen cross-linking techniques, either epi-on, epi-off and different fluence that need to be at least addressed. 


\section{Clinical Need (e.g. Target Patients, Incidence and/or Prevalence of Clinical Need, Total Amount Spent Per Year to Address this Clinical Problem and Target Segment of Market)}

Our experience is suggestive of a significantly higher incidence of keratoconus in the Southern European countries and specifically the Eastern Mediterranean region. The specific incidence in Greece may be especially high. We have additionally noted clinically that there may be a much higher familiar correlation of the disease than the degree that it has been previously reported and considered. We have noted keratoconus signs in the topographic and/or tomographic imaging of at least $80 \%$ of one of the two parents of diagnosed young Greek and/or Cypriot keratoconic patients.

Our team has been pioneering keratoconus screening and cross-linking interventions to arrest progressive keratoconus for more than 10 years, ${ }^{33-38}$ and such activities may occupy more than $50 \%$ of our clinical, surgical and research activities.

Our work attempts to correlate existing diagnostic criteria of keratoconus and cross-linking efficacy with newer and sensitive criteria, such as the overall epithelial thickness, the asymmetry of the epithelial thickness indices, all obtained with anterior segment (AS) OCT, the possibility that the Cassini (multicolored LED-point reflection topographer), who studies elevation data, may be more sensitive in identifying corneal asymmetry in keratoconus and possibly progression, and how these indices correlate with standard Scheimpflug and Placido disk-derived asymmetry indices, as well as with visual acuity and contrast sensitivity data in these patients. Furthermore, biomechanical criteria, of whether there is a substantial benchmark in utilizing the biomechanical response to air puff of normal, keratoconic, and suspect corneas, in the diagnosis and progression of keratoconus, as well as corneal collagen cross-linking in the evaluation of the cross-link effects.

On the contrary, our investigation is suggestive that there may be novel indicators provided by modern corneal imaging devices providing more specific and sensitive indicators for keratoconus assessment. Examples may be found in topographic irregularity indices provided by Scheimpflug imaging and Placido topography, such as the index of surface variance (ISV) and the index of height decentration (IHD). Our investigation carried so far suggests that a better approach may be the examination of such quantitative indicators that reflect the anterior-surface variance across the cornea. These anterior shape-based indices provide positive correlation with topographic keratoconus classification (TKC), based in the traditional Krumeich-Amsler criteria, and may provide a quantitative tool for keratoconus classification and progression assessment.

Specifically, the average coefficient of determination (r2), between ISV and the determined TKC keratoconus severity grade had an average value of 0.793 , and between IHD and TKC, 0.716 respectively. In other words, our initial investigation indicates that there is a significant correlation between the two anterior-surface irregularity indices and keratoconus classification.

Other modalities may also play a role, which will be extensively investigated. A large number of the standard and novel diagnostic modalities that will be incorporated in the study, which are already part of our Institution's screening/ diagnostic inventory and they include the following:

a. Placido topography (Keratograph 5, Oculus, Germany).

b. High-resolution Scheimpflug imaging (Pentacam HR, Oculus, Germany).

c. Novel multicolored-spot reflection topography (Cassini, I-Optics, the Netherlands).

d. Anterior-segment optical coherence tomography offering corneal and epithelial thickness mapping and total corneal refractive power (RtVue, Optovue, USA).

e. Advanced AutoRefractor (Nidek, Japan),

f. Visual acuity/contrast sensitivity/contrast acuity-monocular and binocular (Functional Vision Analyzer, Stereo Optical, USA).

These most modern screening and diagnostic devices offer a large variety of novel keratoconus-specific indicators which have not been studied in such an extent over such a large pool of patients. Specifically, each screening modality offers the following indicators that we investigate:

a. The Keratograph 5 is an advanced placido disk-based corneal topographer with a built-in real keratometer and a color camera. In addition to standard keratometry, the device is capable of examining in a noninvasive function the tear film break-up time and the tear meniscus height measurement and evaluate the lipid layer. Similarly to the Pentacam HR, the device offers the seven specific anterior surface irregularity indices that describe cornea irregularity, specifically the ISV, and the IHD. Our team has extensive record on VideoKeratoscopy-based screening of keratoconus as early as 1996 . 
b. The Pentacam HR is a high-resolution rotating Scheimpflug camera system for AS analysis. The device measures anterior and posterior corneal topography and elevation maps, total corneal refractive power, corneal power distribution, as well as corneal and crystalline lens optical opacities. Specific to keratoconus investigation, the device offers the Belin/ Ambrosio Enhanced Ecstasia, reports. In addition, the device offers values for the following parameters: anterior and posterior keratometry, simulated keratometry, anterior and posterior elevation, and most importantly, seven specific anterior-surface irregularity indices that describe cornea irregularity. Of these seven, two indices have been identified by our previous research as best correlates to keratoconus topographic classification, namely the ISV and the IHD.

c. The Cassini, a newly-introduced elevation-based topographer operating on the principle of multicolored (LED) spotreflection topography, offers, in addition to the standard topometric maps (axial and tangential curvature, anterior elevation, and refractive power), offers anterior surface aberrations measurements, as well as posterior curvature and elevation measurements - a unique feature among topographers. Moreover, several keratoconus-specific data are included, such as the Klyce indices of SAI and SRI.

d. The RtVue-100, novel Fourier-domain anterior-segment OCT device, is unique among similar devices for its offering of not only corneal thickness maps, but corneal epithelial thickness maps covering a 6 mm wide corneal area. The screening by such a device offers specific distinguishing corneal pachymetric features in the diagnosis of keratoconus, namely the asymmetry and focal thinning manifested inferotemporally; quantification of this characteristic pattern may be useful in keratoconus diagnosis and ectasia risk assessment. Four quantitative parameters calculated by AS-OCT pachymetry have been proposed for keratoconus diagnosis. ${ }^{9}$ Specifically, these indices are: (a) S-I: the average thickness of the superior octant minus the average thickness of the inferior, (b) SN-IT: the average superior-nasal octant thickness minus the average of the inferior temporal thickness, (c) min-med: the focal thinning, defined as the difference of the minimum minus the median corneal thickness, and (d) min-max: the thickness range, or global thinning, defined as the minimum minus the maximum corneal thickness.

Specifically to the epithelial thickness imaging, the examination report may offer several keratoconus specific indices, namely the overall epithelial thickness, the epithelial thickness topographic variability and the epithelial thickness range (minimum-maximum). Our team has investigated these parameters prior to the advent of OCT in the realm of epithelial thickness imaging by another unique devices, based on very high frequency scanning ultrasound, the Artemis (Ultralink LLC, Canada). In this study, for the first time, our group showed that an overall thicker epithelium in a specific age group of young keratoconic patients, in addition to the accentuated epithelial thickness range and topographic variability.

\section{Biomechanical Cross-linking Intervention Effects Assessment (in vivo and ex vivo)}

Corneal strength visualization (CorVis ST, Oculus, Germany). The CorVis ST is a functional in vivo corneal biomechanics analyzer employing a noncontact tonometer and enabling recording the corneal reaction to an air impulse. An incorporated high-speed Scheimpflug camera (4,330 frames/sec) records the movements of the cornea which then are displayed on the built-in control. The device enables assessment of corneal properties (corneal hysteresis, an indication of viscous damping in the cornea, reflecting the capacity of corneal tissue to absorb and dissipate energy, and corneal resistance factor, an indicator of the overall resistance of the cornea) for various applications of keratoconus screening and cross-linking assessment.

BioTester 5000 (Cell Scale, Waterloo, Ontario, Canada). The BioTester is a specifically developed biomaterials biaxial strength analyzer for ex vivo corneal rigidity (Young's modulus) measurements within a temperature controlled media bath. The biaxial test system captures and graphically displays live time, force and synchronized video images for results analysis and verification. Data are easily exported to standard spreadsheet programs. Two high-performance actuators (two per axis) are capable of positional resolution for accurate test motion, with inline overload-protected load cell on each axis and a high-resolution digital internal camera to collect time synchronized images for post-test analysis.

We believe that the incorporation of the data described herein will be able to create an algorithm that is more sensitive than the current diffuse criteria employed in keratoconus to not only include topographic asymmetry and thickness asymmetry indices, perhaps more sensitive AS topographic asymmetry indices as well as newly introduced topographic asymmetry indices of epithelial thickness, and differences of the biomechanical response of normal/suspect and keratoconic corneas and the above-mentioned parameters.

The present study aims to further evaluate the postoperative corneal rigidity by the CorVis (Oculus) device, dual axis extensiometry measurements, as well as enzymatic digestion measurements.

1. The hypothesis as far as the diagnosis of keratoconus is that there are more sensitive criteria than the already available criteria for keratoconus are based on topographic asymmetry, thinning and keratometry. We hypothesize that the 
incorporation of data of total epithelial thickness, epithelial asymmetry, as well as biomechanical factors will make the diagnosis of keratoconus far more sensitive and specific, and more importantly the possibility of progression of keratoconus. Obviously, the magnitude and meticulous study of all these parameters proposed in this scientific proposal would be able to accomplish this.

2. The second hypothesis is that there is a different collagen cross-linking effect with different techniques available today either if that involves different fluence, or different riboflavin administration (epi-on/epi-off), or different amounts of total energy applied to cornea. Therefore, our hypothesis is that there is a differential of effect according to the technique employed and it is the intent of this study to develop a benchmark of how each one of these techniques (different protocols) of CXL affects the cornea in vivo and ex vivo and thus create a correlation between them. The fact that the in vivo patient population will be also studied in the fashion that the normal, suspect and keratoconic eyes will be able to provide some data on how the 'disease regression' is accomplished with the above-mentioned techniques.

\section{REFERENCES}

1. Rabinowitz YS. Videokeratographic indices to aid in screening for keratoconus. J Refract Surg 1995 Sep-Oct;11(5):371-379.

2. Klyce SD. Computer-assisted corneal topography. High-resolution graphic presentation and analysis of keratoscopy. Invest Ophthalmol Vis Sci 1984 Dec;25(12):1426-1435.

3. Lopes BT, Ramos IC, Faria-Correia F, Luz A, de Freitas Valbon B, Belin MW, Ambrosio R Jr. Correlation of topometric and tomographic indices with visual acuity in patients with keratoconus. Int J Kerat Ect Cor Dis 2012;1(3):167-172.

4. Kanellopoulos AJ, Moustou V, Asimellis G. Evaluation of visual acuity, pachymetry and anterior-surface irregularity in keratoconus and crosslinking intervention follow-up in 737 cases. Int J Kerat Ect Cor Dis 2013;2(3):95-103.

5. Holladay JT. Keratoconus detection using corneal topography. J Refract Surg 2009 Oct;25(10 Suppl):S958-S962.

6. Randleman JB, Russell B, Ward MA, Thompson KP, Stulting RD. Risk factors and prognosis for corneal ectasia after LASIK. Ophthalmology 2003 Feb;110(2):267-275.

7. Fam HB, Lim KL. Corneal elevation indices in normal and keratoconic eyes. J Cataract Refract Surg 2006 Aug;32(8):1281-1287.

8. Sedghipour MR, Sadigh AL, Motlagh BF. Revisiting corneal topography for the diagnosis of keratoconus: use of Rabinowitz's KISA\% index. Clin Ophthalmol 2012;6:181-184.

9. Wollensak G, Spoerl E, Seiler T. Riboflavin/ultraviolet: a induced collagen crosslinking for the treatment of keratoconus. Am J Ophthalmol 2003 May;135(5):620-627.

10. Sondergaard AP, Ivarsen A, Hjortdal J. Corneal resistance to shear force after UVA-riboflavin cross- linking. Invest Ophthalmol Vis Sci 2013 Jul;54(7):5059-5069.

11. Spoerl E, Wollensak G, Seiler T. Increased resistance of crosslinked cornea against enzymatic digestion. Curr Eye Res 2004 Jul;29(1): $35-40$.

12. Kanellopoulos AJ, Asimellis G. Introduction of quantitative and qualitative cornea optical coherence tomography findings induced by collagen cross-linking for keratoconus: a novel effect measurement benchmark. Clin Ophthalmol 2013;7:329-335.

13. Szalai E, Berta A, Hassan Z, Modis L Jr. Reliability and repeatability of swept-source Fourier-domain optical coherence tomography and Scheimpflug imaging in keratoconus. J Cataract Refract Surg 2012;38(3):485-494.

14. Kanellopoulos AJ, Asimellis G. Comparison of high-resolution Scheimpflug and high-frequency ultrasound biomicroscopy to anteriorsegment OCT corneal thickness measurements. Clin Ophthalmol 2013;7:2239-2247.

15. O’Brart DP, Chan E, Samaras K, Patel P, Shah SP. A randomised, prospective study to investigate the efficacy of riboflavin/ultraviolet A $(370 \mathrm{~nm})$ corneal collagen cross-linkage to halt the progression of keratoconus. Br J Ophthalmol 2011 Nov;95(11):1519-1524.

16. Hersh PS, Greenstein SA, Fry KL. Corneal collagen cross-linking for keratoconus and corneal ectasia: one-year results. J Cataract Refract Surg 2011;37(1):149-160.

17. Wittig-Silva C, Whiting M, Lamoureux E, Lindsay RG, Sullivan LJ, Snibson GR. A randomized controlled trial of corneal collagen cross-linking in progressive keratoconus: preliminary results. J Refract Surg 2008 Sep;24(7):S720-S725.

18. Li X, Yang H, Rabinowitz YS. Keratoconus: classification scheme based on videokeratography and clinical signs. J Cataract Refract Surg 2009 Sep;35(9):1597-1603.

19. Lass JH, Lembach RG, Park SB, Hom DL, Fritz ME, Svilar GM, Nuamah IF, Reinhart WJ, Stocker EG, Keates RH, et al. Clinical management of keratoconus: a multicenter analysis. Ophthalmology 1990 Apr;97(4):433-445.

20. Gore DM, Shortt AJ, Allan BD. New clinical pathways for keratoconus. Eye (Lond) 2013 Mar;27(3):329-339.

21. Kelly TL, Williams KA, Coster DJ. Australian Corneal Graft Registry. Corneal transplantation for keratoconus: a registry study. Arch Ophthalmol 2011 Jun;129(6):691-697.

22. Kelly TL, Coster DJ, Williams KA. Repeat penetrating corneal transplantation in patients with keratoconus. Ophthalmology 2011 Aug;118(8):1538-1542.

23. Reinhart WJ, Musch DC, Jacobs DS, Lee WB, Kaufman SC, Shtein RM. Deep anterior lamellar keratoplasty as an alternative to penetrating keratoplasty: a report by the American Academy of Opthalmology. Ophthalmology 2011 Jan;118(1):209-218.

24. Borderie VM, Sandali O, Bullet J, Gaujoux T, Touzeau O, Laroche L. Long-term results of deep anterior lamellar versus penetrating keratoplasty. Ophthalmology 2012 Feb;119(2):249-255.

25. Vinciguerra P, Albe E, Frueh BE, Trazza S, Epstein D. Two-year corneal cross-linking results in patients younger than 18 years with documented progressive keratoconus. Am J Ophthalmol 2012 Sep;154(3):520-526. 
26. Caporossi A, Mazzotta C, Baiocchi S, Caporossi T. Long-term results of riboflavin ultraviolet a corneal collagen cross-linking for keratoconus in Italy: the Siena eye cross study. Am J Ophthalmol 2010 Apr;149(4):585-593.

27. Koller T, Mrochen M, Seiler T. Complication and failure rates after corneal cross-linking. J Cataract Refract Surg 2009 Aug;35(8):13581362.

28. Rebenitsch RL, Kymes SM, Walline JJ, Gordon MO. The lifetime economic burden of keratoconus: a decision analysis using a Markov model. Am J Ophthalmol 2011 May;151(5):768-773.

29. Colin J, Velou S. Current surgical options for keratoconus. J Cataract Refract Surg 2003 Feb;29(2):379-386.

30. Roe RH, Lass JH, Brown GC, Brown MM. The value-based medicine comparative effectiveness and cost-effectiveness of penetrating keratoplasty for keratoconus. Cornea 2008 Oct;27(9):1001-1007.

31. Koo TS, Finkelstein E, Tan D, Mehta JS. Incremental cost-utility analysis of deep anterior lamellar keratoplasty compared with penetrating keratoplasty for the treatment of keratoconus. Am J Ophthalmol 2011 Jul;152(1):40-47.

32. Yildiz EH, Cohen EJ, Virdi AS, Hammersmith KM, Laibson PR, Rapuano CJ. Quality of life in keratoconus patients after penetrating keratoplasty. Am J Ophthalmol 2010 Mar;149(3):416-422.

33. Kanellopoulos AJ, Binder PS. Collagen cross-linking (CCL) with sequential topography-guided PRK: a temporizing alternative for keratoconus to penetrating keratoplasty. Cornea 2007 Aug;26(7):891-895.

34. Krueger RR, Ramos-Esteban JC, Kanellopoulos AJ. Staged intrastromal delivery of riboflavin with UVA cross-linking in advanced bullous keratopathy: laboratory investigation and first clinical case. J Refract Surg 2008 Sep;24(7):S730-S736.

35. Kanellopoulos AJ. Collagen cross-linking in early keratoconus with riboflavin in a femtosecond laser-created pocket: initial clinical results. J Refract Surg 2009 Nov;25(11):1034-1047.

36. Kanellopoulos AJ. Comparison of sequential vs same-day simultaneous collagen cross-linking and topography-guided PRK for treatment of keratoconus. J Refract Surg 2009 Sep;25(9):S812-S818.

37. Krueger RR, Kanellopoulos AJ. Stability of simultaneous topography-guided photorefractive keratectomy and riboflavin/UVA crosslinking for progressive keratoconus: case reports. J Refract Surg 2010 Oct;26(10):S827-S832.

38. Kanellopoulos AJ, Binder PS. Management of corneal ectasia after LASIK with combined, same-day, topography-guided partial transepithelial PRK and collagen cross-linking: the athens protocol. J Refract Surg 2011 May;27(5):323-331.

A John Kanellopoulos MD

Clinical Professor, Department of Ophthalmology

NYU School of Medicine, New York, USA 